FACTA UNIVERSITATIS (NIŠ)

Ser. Math. Inform. Vol. 31, No 5 (2016), 1091-1106

DOI:10.22190/FUMI1605091A

\title{
SUPRA HOMEOMORPHISM IN SUPRA TOPOLOGICAL ORDERED SPACES
}

\author{
Mohamed Abo-elhamayel and Tareq M. Al-shami
}

\begin{abstract}
The purpose of this paper is to introduce the concepts of x-supra continuous (open, closed, homeomorphism) maps in supra topological ordered spaces for $\mathrm{x} \in$ $\{\mathrm{I}, \mathrm{D}, \mathrm{B}\}$. We study the relationship among these types with the help of examples and investigate the equivalent conditions for each concept. In particular, we present the sufficient conditions for maps to preserve some of separation axioms.
\end{abstract}

Keywords: Topological ordered spaces; Supra continuous maps; Separation axioms.

\section{Introduction and Preliminaries}

Nachbin [7] in 1965, started the study of topological ordered space $(X, \tau, \preceq)$, which consists of a topological space equipped with a partial relation. In 1968, McCartan [6] studied separation axioms in topological ordered spaces by utilizing monotone neighborhoods. In 1983, Mashhour et al. [5] introduced the concept of supra topological spaces and generalized some properties of topological spaces to supra topological spaces such as continuity and some separation axioms. In 2002, Kumar [4] introduced the concept of homeomorphism maps in supra topological ordered spaces. He generalized the properties of continuous (open, closed, homeomorphism) maps in topological spaces and obtained interesting properties. In 2004, Das [1] introduced ordered separation axioms in supra topological spaces by generalized results which reached by McCartan [6]. In 2012, Rao and Chudamani ([8], [9], [10], [11]) introduced and studied semi-homeomorphism (pre-homeomorphism, $\alpha$ homeomorphism, $\beta$-homeomorphism) maps in topological ordered spaces. In 2015, El-Shafei et al.[3] introduced new separation axioms in supra topological ordered spaces. The aim of the present paper is to established the concepts of $\mathrm{x}$-supra continuous (open, closed, homeomorphism) maps in supra topological ordered spaces for $\mathrm{x} \in\{\mathrm{I}, \mathrm{D}, \mathrm{B}\}$. Also, we investigate necessary and sufficient conditions for these maps to preserve some ordered separation axioms. Many of the findings that raised

Received June 21, 2016; accepted August 06, 2016

2010 Mathematics Subject Classification. Primary 54F05; Secondary 54D10, 54A99 
at are generalizations of those findings in topological ordered spaces. Illustrative examples are included to point out the relationships among these concepts. Throughout this paper, A triple $(X, \tau, \preceq)$ where $\tau$ is a topology (supra topology) on $X$ and $\preceq$ is a partial order relation on $X$, is said to be a topological (supra topological) ordered space. If $(X, \preceq)$ is a partially ordered set (poset, for short), then for each $a \in X, i(a)=\{b \in X: a \preceq b\}$ and $d(a)=\{b \in X: b \preceq a\}$. If $A \subseteq X$, then $i(A)=\bigcup\{i(a): a \in A\}$ and $d(A)=\bigcup\{d(a): a \in A\}$. If $A=i(A)$ (resp. $A=d(A), A=d(A)=i(A))$, then $A$ is called an increasing (resp. a decreasing, a balancing) set or simply I (resp. D,B)-set. The diagonal relation is denoted by $\triangle$.

Definition 1.1. [3] A subset $G$ of a supra topological space $(X, \tau)$ is called:

(i) I-supra open if $G$ is supra open and increasing.

(ii) D-supra open if $G$ is supra open and decreasing.

(iii) B-supra open if $G$ is supra open and balancing.

Definition 1.2. [3] A supra topological ordered space $(X, \mu, \preceq)$ is called:

(i) Strong lower supra $T_{1}$-ordered space if for each $a, b \in X$ such that $a \npreceq b$, there exists a decreasing supra open set $G$ containing $b$ to which $a \notin G$.

(ii) Strong Upper supra $T_{1}$-ordered space if for each $a, b \in X$ such that $a \npreceq b$, there exists an increasing supra open set $G$ containing $a$ to which $b \notin G$.

(iii) Strong supra $T_{0}$-ordered space if it is Strong lower supra $T_{1}$-ordered or strong upper supra $T_{1}$-ordered.

(iv) Strong supra $T_{1}$-ordered space if it is both strong lower supra $T_{1}$-ordered and strong upper supra $T_{1}$-ordered.

(v) Strong supra $T_{2}$-ordered if for each $a, b \in X$ such that $a \npreceq b$, there exist disjoint supra open sets $G$ and $H$ containing $a$ and $b$ respectively such that $G$ is increasing and $H$ is decreasing.

Definition 1.3. [5] Consider $(X, \tau)$ is a supra topological space and $(Y, \theta)$ is a topological space. A map $f: X \rightarrow Y$ is called supra continuous if the inverse image of each open set in $Y$ is a supra open set in $X$.

A map $f$ of $\left(X, \preceq_{1}\right)$ into $\left(Y, \preceq_{2}\right)$ is called order preserving (or increasing) if for each $a, b \in X$ such that $a \preceq_{1} b$, then $f(a) \preceq_{2} f(b)$. A map $f$ of $\left(X, \preceq_{1}\right)$ into $\left(Y, \preceq_{2}\right)$ is called order embedding if for each $a, b \in X$, then $a \preceq_{1} b$ iff $f(a) \preceq_{2} f(b)$. 


\section{Supra continuous maps in supra topological ordered spaces}

In this section, we introduce the concepts of I-supra continuous, D-supra continuous and B-supra continuous maps in supra topological ordered spaces and discus their important properties, especially with respect to the sufficient conditions for some maps to preserve some separation axioms.

Definition 2.1. Consider $\left(X, \tau, \preceq_{1}\right)$ is a supra topological ordered space and $\left(Y, \theta, \preceq_{2}\right.$ ) is a topological ordered space. A map $f: X \rightarrow Y$ is said to be I-supra (resp. Dsupra, B-supra) continuous if the inverse image of each open set in $Y$ is an I-supra (resp. a D-supra, a B-supra) open set in $X$.

Remark 2.1. (i) Every I-supra (D-supra, B-supra) continuous map is supra continuous.

(ii) Every B-supra continuous map is I-supra continuous and D-supra continuous.

The following two examples illustrate that a supra continuous map (resp. Isupra continuous) need not be I-supra continuous or D-supra continuous or B-supra continuous (resp. B-supra continuous) map.

Example 2.1. Let the supra topology $\mu=\{\varnothing, X,\{a, b\},\{a, c\}\}$ on $X=\{a, b, c\}$, the topology $\theta=\{\varnothing, Y,\{x, y\}\}$ on $Y=\{x, y, z\}$, and the partial relation $\preceq_{1}=\triangle \bigcup\{(a, b),(b, c)$, $(a, c)\}$ on $X$. The map $f: X \rightarrow Y$ is defined as follows $f(a)=x, f(b)=z, f(c)=y$. Obviously, $f$ is supra continuous. Now, $\{x, y\}$ is an open subset of $Y$, but $f^{-1}(\{x, y\})=\{a, c\}$ which is neither a decreasing nor an increasing supra open subset of $X$. Then $f$ is not $\mathrm{I}(\mathrm{D}, \mathrm{B})$-supra continuous.

Example 2.2. We replace only the partial order relation in Example 2.1 by $\preceq=\triangle \bigcup\{(b, a)\}$. Then the map $f$ is I-supra continuous, but not B-supra continuous.

The relationships among the types of supra continuous maps are illustrated in the following Figure.

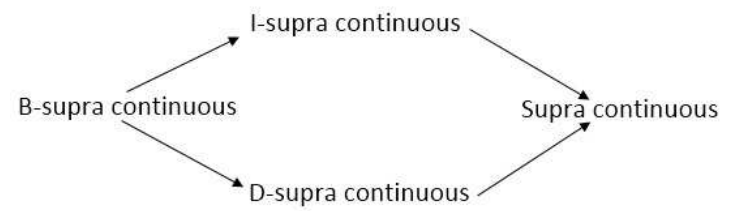

FIG. 2.1: The relationships among types of supra continuous maps

Definition 2.2. Consider $\left(X, \tau, \preceq_{1}\right)$ is a supra topological ordered space and $\left(Y, \theta, \preceq_{2}\right.$ ) is a topological ordered space. A map $f: X \rightarrow Y$ is called I-supra (resp. D-supra, B-supra) continuous at $p \in X$ if the inverse image of each open set containing $f(p)$ is an I-supra (resp. a D-supra, a B-supra) open set containing $p$. 
Theorem 2.1. A map $f:\left(X, \tau, \preceq_{1}\right) \rightarrow\left(Y, \theta, \preceq_{2}\right)$ is $I$ (resp. D, B)-supra continuous iff it is I-supra (resp. D-supra, B-supra) continuous at each $p \in X$.

Proof. In the case of $f$ is I-supra continuous.

$'{ }^{\prime}$ It is clear.

$' \Leftarrow{ }^{\prime}$ Let $G$ be an open subset of $Y$. Then we have two cases:

(i) $f^{-1}(G)=\varnothing$ which is an I-supra open subset of $X$.

(ii) $f^{-1}(G) \neq \varnothing$, then there exists $p \in X$ such that $f(p) \in G$. Therefore $p \in$ $f^{-1}(G)$ which is an I-supra open subset of $X$. Since we choose $G$ and $p$ arbitrary, then $f$ is I-supra continuous.

A similar proof can be given for the cases between parentheses.

Definition 2.3. Let $(X, \tau, \preceq)$ be a supra topological ordered space and $E$ be a subset of $X$. Then

$E^{i s o}=\bigcup\{G: G$ is an I-supra open set included in $E\}$, $E^{d s o}=\bigcup\{G: G$ is a D-supra open set included in $E\}$,

$E^{b s o}=\bigcup\{G: G$ is a B-supra open set included in $E\}$, $E^{i s c l}=\bigcap\{H: H$ is an I-supra closed set including $E\}$, $E^{d s c l}=\bigcap\{H: H$ is a D-supra closed set including $E\}$, $E^{b s c l}=\bigcap\{H: H$ is a B-supra closed set including $E\}$.

Lemma 2.1. Let $(X, \mu, \preceq)$ be a supra topological ordered space and $E$ be a subset of $X$. Then:

(i) $\left((E)^{d s c l}\right)^{c}=\left((E)^{c}\right)^{i s o}$.

(ii) $\left((E)^{i s c l}\right)^{c}=\left((E)^{c}\right)^{d s o}$.

(iii) $\left((E)^{b s c l}\right)^{c}=\left((E)^{c}\right)^{b s o}$.

Proof. (i) $\left((E)^{d s c l}\right)^{c}=\{\cap F: F \text { is a D-supra closed including } E\}^{c}$ $=\cup\left\{F^{c}: F^{c}\right.$ is an I-supra open included in $\left.E^{c}\right\}=\left((E)^{c}\right)^{i s o}$.

The proof of (ii) and (iii) is similar to (i).

Theorem 2.2. For a map $g:\left(X, \tau, \preceq_{1}\right) \rightarrow\left(Y, \theta, \preceq_{2}\right)$, where $\left(X, \tau, \preceq_{1}\right)$ is a supra topological ordered space and $\left(Y, \theta, \preceq_{2}\right)$ is a topological ordered space, the following statements are equivalent:

(i) $g$ is I-supra continuous;

(ii) The inverse image of each closed subset of $Y$ is a D-supra closed subset of $X$;

(iii) $\left(g^{-1}(H)\right)^{d s c l} \subseteq g^{-1}(\operatorname{cl}(H))$, for every $H \subseteq Y$; 
(iv) $g\left(A^{d s c l}\right) \subseteq c l(g(A))$, for every $A \subseteq X$;

(v) $g^{-1}(\operatorname{int}(H)) \subseteq\left(g^{-1}(H)\right)^{\text {iso }}$, for every $H \subseteq Y$.

Proof. (i) $\Rightarrow$ (ii) Consider $H$ is a closed subset of $Y$. Then $H^{c}$ is open. Therefore $g^{-1}\left(H^{c}\right)=\left(g^{-1}(H)\right)^{c}$ is an I-supra open subset of $X$. Thus $g^{-1}(H)$ is a D-supra closed.

(ii) $\Rightarrow$ (iii) For any subset $H$ of $Y, \operatorname{cl}(H)$ is a closed subset of $Y$. Since $g^{-1}(c l(H))$ is a D-supra closed subset of $X$, then $\left(g^{-1}(H)\right)^{d s c l} \subseteq\left(g^{-1}(c l(H))^{d s c l}=g^{-1}(c l(H))\right.$. (iii) $\Rightarrow$ (iv): Consider $A$ is any subset of $X$. Then $A^{d s c l} \subseteq\left(g^{-1}(g(A))^{d s c l} \subseteq\right.$ $g^{-1}\left(\operatorname{cl}(g(A))\right.$. Thus $g\left(A^{d s c l}\right) \subseteq g\left(g^{-1}(\operatorname{cl}(g(A))) \subseteq \operatorname{cl}(g(A))\right.$.

(iv) $\Rightarrow(\mathbf{v})$ : Let $H$ be any subset of $Y$. Then $g\left(X-\left(g^{-1}(H)\right)^{i s o}\right)=g\left(\left(\left(g^{-1}(H)\right)^{c}\right)^{d s c l}\right)$ $\subseteq \operatorname{cl}\left(g\left(g^{-1}(H)\right)^{c}\right)=\operatorname{cl}\left(g\left(g^{-1}\left(H^{c}\right)\right)\right) \subseteq \operatorname{cl}(Y-H)=Y-i n t(H)$. Therefore $(X-$ $\left.\left(g^{-1}(H)\right)^{i s o}\right) \subseteq g^{-1}(Y-\operatorname{int}(H))=X-g^{-1}(\operatorname{int}(H))$. Thus $g^{-1}(\operatorname{int}(H)) \subseteq\left(g^{-1}(H)\right)^{i s o}$. (v) $\Rightarrow$ (i) Consider $H$ is an open subset of $Y$. Then $g^{-1}(H)=g^{-1}(\operatorname{int}(H)) \subseteq$ $\left(g^{-1}(H)\right)^{i s o}$, but $\left(g^{-1}(H)\right)^{i s o} \subseteq g^{-1}(H)$. Therefore $g^{-1}(H)$ is an I-supra open subset of $X$. Thus $g$ is I-supra continuous.

Theorem 2.3. For a map $g:\left(X, \tau, \preceq_{1}\right) \rightarrow\left(Y, \theta, \preceq_{2}\right)$, where $\left(X, \tau, \preceq_{1}\right)$ is a supra topological ordered space and $\left(Y, \theta, \preceq_{2}\right)$ is a topological ordered space, the following statements are equivalent:

(i) $g$ is D-supra continuous;

(ii) The inverse image of each closed subset of $Y$ is an I-supra closed subset of $X$;

(iii) $\left(g^{-1}(H)\right)^{i s c l} \subseteq g^{-1}(c l(H))$, for every $H \subseteq Y$;

(iv) $g\left(A^{i s c l}\right) \subseteq \operatorname{cl}(g(A))$, for every $A \subseteq X$;

(v) $g^{-1}(\operatorname{int}(H)) \subseteq\left(g^{-1}(H)\right)^{d s o}$, for every $H \subseteq Y$.

Proof. The proof is similar to that of Theorem 2.2.

Theorem 2.4. For a map $g:\left(X, \tau, \preceq_{1}\right) \rightarrow\left(Y, \theta, \preceq_{2}\right)$, where $\left(X, \tau, \preceq_{1}\right)$ is a supra topological ordered space and $\left(Y, \theta, \preceq_{2}\right)$ is a topological ordered space, the following statements are equivalent:

(i) $g$ is B-supra continuous;

(ii) The inverse image of each closed subset of $Y$ is a B-supra closed subset of $X$;

(iii) $\left(g^{-1}(H)\right)^{b s c l} \subseteq g^{-1}(c l(H))$, for every $H \subseteq Y$;

(iv) $g\left(A^{b s c l}\right) \subseteq \operatorname{cl}(g(A))$, for every $A \subseteq X$;

(v) $g^{-1}(\operatorname{int}(H)) \subseteq\left(g^{-1}(H)\right)^{\text {bso }}$, for every $H \subseteq Y$.

Proof. The proof is similar to that of Theorem 2.2. 
Theorem 2.5. Consider $\left(X, \tau, \preceq_{1}\right)$ is a supra topological ordered space and $\left(Y, \theta, \preceq_{2}\right.$ ) is a topological ordered space. Let a bijective map $f: X \rightarrow Y$ be I-supra continuous and $f^{-1}$ be an order preserving map. If $\left(Y, \theta, \preceq_{2}\right)$ is a lower $T_{1}$-ordered space, then $\left(X, \tau, \preceq_{1}\right)$ is a strong lower supra $T_{1}$-ordered space.

Proof. Let $a, b \in X$ such that $a \npreceq_{1} b$. Then there exist $x, y \in Y$ such that $x=$ $f(a), y=f(b)$. Since $f^{-1}$ is an order preserving map, then $x \npreceq_{2} y$. Since $\left(Y, \theta, \preceq_{2}\right)$ is a lower $T_{1}$-ordered space, then there exists an increasing neighborhood $W$ of $x$ in $Y$ such that $x \in W$ and $y \notin W$. Therefore there exists an open set $G$ such that $x \in G \subseteq W$. Since $f$ is bijective I-supra continuous, then $a \in f^{-1}(G)$ which is I-supra open and $b \notin f^{-1}(G)$. Thus $\left(X, \tau, \preceq_{1}\right)$ is a strong lower supra $T_{1}$-ordered space.

Theorem 2.6. Consider $\left(X, \tau, \preceq_{1}\right)$ is a supra topological ordered space and $\left(Y, \theta, \preceq_{2}\right.$ ) is a topological ordered space. Let a bijective map $f: X \rightarrow Y$ be D-supra continuous and $f^{-1}$ be an order preserving map. If $\left(Y, \theta, \preceq_{2}\right)$ is an upper $T_{1}$-ordered space, then $\left(X, \tau, \preceq_{1}\right)$ is a strong upper supra $T_{1}$-ordered space.

Proof. The proof is similar to that of Theorem 2.5.

Theorem 2.7. Consider $\left(X, \tau, \preceq_{1}\right)$ is a supra topological ordered space and $\left(Y, \theta, \preceq_{2}\right.$ ) is a topological ordered space. Let a bijective map $f: X \rightarrow Y$ be B-supra continuous and $f^{-1}$ be an order preserving map. If $\left(Y, \theta, \preceq_{2}\right)$ is a $T_{i}$-ordered space, then $\left(X, \tau, \preceq_{1}\right)$ is a strong supra $T_{i}$-ordered space for $i=0,1,2$.

Proof. In the case of $i=2$. Let $a, b \in X$ such that $a \npreceq_{1} b$. Then there exist $x, y \in Y$ such that $x=f(a), y=f(b)$. Since $f^{-1}$ is an order preserving map, then $x \npreceq_{2} y$. Since $\left(Y, \theta, \preceq_{2}\right)$ is a $T_{2}$-ordered space, then there exist disjoint neighborhoods $W_{1}$ and $W_{2}$ of $x$ and $y$ respectively such that $W_{1}$ is increasing and $W_{2}$ is decreasing. Therefore there are disjoint open sets $G$ and $H$ containing $x$ and $y$, respectively. Since $f$ is bijective B-supra continuous, then $a \in f^{-1}(G)$ which is an increasing supra open subset of $X, b \in f^{-1}(H)$ which is a decreasing supra open subset of $X$ and $f^{-1}(G) \bigcap f^{-1}(H)=\varnothing$. Thus $\left(X, \tau, \preceq_{1}\right)$ is a strong supra $T_{2}$-ordered space.

In a similar way, we can prove theorem in the cases of $i=0,1$.

Theorem 2.8. Consider $\left(X, \tau, \preceq_{1}\right)$ is a supra topological ordered space, $\left(Y, \theta, \preceq_{2}\right)$ is a topological ordered space and $f: X \rightarrow Y$ is a bijective supra continuous map such that $f$ is ordered embedding. If $\left(Y, \theta, \preceq_{2}\right)$ is a $T_{i}$-ordered space (a strong $T_{i}$ ordered space), then $\left(X, \tau, \preceq_{1}\right)$ is a supra $T_{i}$-ordered space (a strong supra $T_{i}$-ordered space) for $i=0,1,2$.

Proof. In the case of $i=2$. Let $a, b \in X$ such that $a \npreceq_{1} b$. Then there exist $x, y \in Y$ such that $x=f(a), y=f(b)$. Since $f$ is ordered embedding, then $x \npreceq_{2} y$. Since $\left(Y, \theta, \preceq_{2}\right)$ is a $T_{2}$-ordered space, then there exist disjoint neighborhoods $W_{1}$ and $W_{2}$ of $x$ and $y$ respectively such that $W_{1}$ is increasing and $W_{2}$ is decreasing. Therefore 
there are disjoint open sets $G$ and $H$ containing $x$ and $y$, respectively. Since $f$ is bijective supra continuous and order preserving, then $f^{-1}(G) \subseteq f^{-1}\left(W_{1}\right)$ which is an increasing supra neighborhood of $a, f^{-1}(H) \subseteq f^{-1}\left(W_{2}\right)$ which is a decreasing supra neighborhood of $b$ and $f^{-1}\left(W_{1}\right) \bigcap f^{-1}\left(W_{2}\right)=\varnothing$. Thus $\left(X, \tau, \preceq_{1}\right)$ is a supra $T_{2}$-ordered space.

Similarly, one can prove theorem in the cases of $i=0,1$.

A similar proof can be given for the case between parentheses.

Theorem 2.9. Consider $\left(X, \tau, \preceq_{1}\right)$ is a supra topological ordered space, $\left(Y, \theta, \preceq_{2}\right)$ is a topological ordered space and $f: X \rightarrow Y$ is an injective B-supra continuous map. If $\left(Y, \theta, \preceq_{2}\right)$ is a $T_{i}$-space, then $\left(X, \tau, \preceq_{1}\right)$ is a strong supra $T_{i}$-ordered space for $i=1,2$.

Proof. We prove theorem when $i=2$ and the other case is similar. Let $a, b \in X$ such that $a \npreceq_{1} b$. Then there exist $x, y \in Y$ such that $f(a)=x, f(b)=y$ and $x \neq y$. Since $\left(Y, \theta, \preceq_{2}\right)$ is a $T_{2}$-space, then there exist disjoint open sets $G$ and $H$ such that $x \in G$ and $y \in H$. Therefore $a \in f^{-1}(G)$ which is an increasing supra open subset of $X, b \in f^{-1}(H)$ which is a decreasing supra open subset of $X$ and $f^{-1}(G) \bigcap f^{-1}(H)=\varnothing$. Thus $\left(X, \tau, \preceq_{1}\right)$ is a strong supra $T_{2}$-ordered space.

\section{Supra open (closed) maps in supra topological ordered spaces}

In this section, we introduce the concepts of I-supra open (closed), D-supra open (closed) and B-supra open (closed) maps in supra topological ordered space. We study and investigate their main properties in detail. Also, we point out relationships among them and with $\mathrm{x}$-supra continuous, for $\mathrm{x} \in\{\mathrm{I}, \mathrm{D}, \mathrm{B}\}$

Definition 3.1. Consider $(X, \tau)$ is a topological space and $(Y, \theta)$ is a supra topological space. A map $g: X \rightarrow Y$ is said to be supra open (resp. supra closed) if the image of any open (resp. closed) subset of $X$ is a supra open (resp. supra closed) subset of $Y$.

Definition 3.2. Consider $\left(X, \tau, \preceq_{1}\right)$ is a topological ordered space and $\left(Y, \theta, \preceq_{2}\right)$ is a supra topological ordered space. A map $g: X \rightarrow Y$ is said to be

(i) I-supra (resp. D-supra, B-supra) open if the image of any open subset of $X$ is an I-supra (resp. a D-supra, a B-supra) open subset of $Y$.

(ii) I-supra (resp. D-supra, B-supra) closed if the image of any closed subset of $X$ is an I-supra (resp. a D-supra, a B-supra) closed subset of $Y$.

Remark 3.1. (i) Every I-supra (D-supra, B-supra) open map is supra open.

(ii) Every I-supra (D-supra, B-supra) closed map is supra closed.

(iii) Every B-supra open (resp. B-supra closed) map is I-supra open and D-supra open (resp. I-supra closed and D-supra closed). 
The following two examples illustrate that a supra open (resp. D-supra open) map need not be I-supra open or D-supra open or B-supra open (resp. B-supra open) map.

Example 3.1. Let the topologies $\tau=\theta=\{\varnothing, X,\{a\}\{a, b\}\}$ on $X=Y=\{a, b, c\}$, the supra topology associated with $\theta$ be $\theta^{\star}=\{\varnothing, X,\{a\}\{a, b\},\{b, c\}\}$ and the partial relation $\preceq_{2}=\triangle \bigcup\{(b, a),(a, c),(b, c)\}$ on $Y$. The map $f: X \rightarrow Y$ is defined as follows $f(a)=f(b)=a, f(c)=b$. Obviously, $f$ is a supra open map. Now, $\{a\}$ is an open subset of $X$, but $f(\{a\})=\{a\}$ which is neither a decreasing nor an increasing supra open subset of $Y$. Then $f$ is not $\mathrm{I}(\mathrm{D}, \mathrm{B})$-supra open.

Example 3.2. We replace only the partial order relation in Example 3.1 by $\preceq=\triangle \bigcup\{(a, c)$, $(b, c)\}$. Then the map $f$ is D-supra open, but is not B-supra open map.

The following two examples illustrate that a supra closed (resp. an I-supra closed) map need not be I-supra closed or D-supra closed or B-supra closed (resp. a B-supra closed) map.

Example 3.3. Let the topologies $\tau=\theta=\{\varnothing, X,\{b, c\}\}$ on $X=Y=\{a, b, c\}$, the supra topology associated with $\theta$ be $\mu=\{\varnothing, X,\{a, b\},\{b, c\}\}$ and the partial relation $\preceq_{2}=\triangle \bigcup\{(b, c),(c, a),(b, a)\}$ on $Y$. The map $f: X \rightarrow Y$ is defined as follows $f(a)=$ $f(c)=c, f(b)=b$. Obviously, $f$ is a supra closed map. Now, $\{a\}$ is a closed subset of $X$, but $f(\{a\})=\{c\}$ is neither a decreasing nor an increasing supra closed subset of $Y$. Then $f$ is not $\mathrm{I}(\mathrm{D}, \mathrm{B})$-supra closed.

Example 3.4. We replace only the partial order relation in Example 3.3 by $\preceq=\triangle \bigcup\{(b, c)\}$. Then the map $f$ is I-supra closed, but is not B-supra closed.

The relationships among types of supra open (supra closed) maps are illustrated in the following Figure.

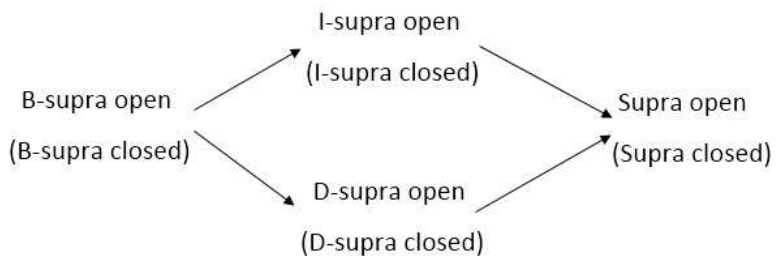

FIG. 3.1: The relationships among types of supra closed maps

Theorem 3.1. For a map $f:\left(X, \tau, \preceq_{1}\right) \rightarrow\left(Y, \theta, \preceq_{2}\right)$, where $\left(X, \tau, \preceq_{1}\right)$ is a topological ordered space and $\left(Y, \theta, \preceq_{2}\right)$ is a supra topological ordered space, the following statements are equivalent:

(i) $f$ is I-supra open; 
(ii) $\operatorname{int}\left(f^{-1}(H)\right) \subseteq f^{-1}\left(H^{i s o}\right)$, for every $H \subseteq Y$;

(iii) $f(\operatorname{int}(G)) \subseteq(f(G))^{\text {iso }}$, for every $G \subseteq X$.

Proof. (i) $\Rightarrow$ (ii) : Since $\operatorname{int}\left(f^{-1}(H)\right)$ is an open subset of $X$, then $f\left(\operatorname{int}\left(f^{-1}(H)\right)\right)$ is an I-supra open subset of $Y$. Since $f\left(\operatorname{int}\left(f^{-1}(H)\right)\right) \subseteq f\left(f^{-1}(H)\right) \subseteq H$, then $\operatorname{int}\left(f^{-1}(H)\right) \subseteq f^{-1}\left(H^{i s o}\right)$.

(ii) $\Rightarrow$ (iii) : Replacing $H$ by $f(G)$ in (ii), we obtain int $\left(f^{-1}(f(G))\right) \subseteq f^{-1}\left((f(G))^{i s o}\right)$. Since $\operatorname{int}(G) \subseteq f^{-1}\left(f\left(\operatorname{int}\left(f^{-1}(f(G))\right)\right)\right) \subseteq f^{-1}\left((f(G))^{\text {iso }}\right)$, then $f(\operatorname{int}(G)) \subseteq(f(G))^{i s o}$. (iii) $\Rightarrow$ (i) : Let $G$ be an open subset of $X$. Then $f(\operatorname{int}(G))=f(G) \subseteq(f(G))^{i s o}$. Thus $f$ is an I-supra open map.

In a similar way one can prove the following two theorems.

Theorem 3.2. For a map $f:\left(X, \tau, \preceq_{1}\right) \rightarrow\left(Y, \theta, \preceq_{2}\right)$, where $\left(X, \tau, \preceq_{1}\right)$ is a topological ordered space and $\left(Y, \theta, \preceq_{2}\right)$ is a supra topological ordered space, the following statements are equivalent:

(i) $f$ is D-supra open;

(ii) $\operatorname{int}\left(f^{-1}(H)\right) \subseteq f^{-1}\left(H^{d s o}\right)$, for every $H \subseteq Y$;

(iii) $f(\operatorname{int}(G)) \subseteq(f(G))^{d s o}$, for every $G \subseteq X$.

Theorem 3.3. For a map $f:\left(X, \tau, \preceq_{1}\right) \rightarrow\left(Y, \theta, \preceq_{2}\right)$, where $\left(X, \tau, \preceq_{1}\right)$ is a topological ordered space and $\left(Y, \theta, \preceq_{2}\right)$ is a supra topological ordered space, the following statements are equivalent:

(i) $f$ is B-supra open;

(ii) $\operatorname{int}\left(f^{-1}(H)\right) \subseteq f^{-1}\left(H^{\text {bso }}\right)$, for every $H \subseteq Y$;

(iii) $f(\operatorname{int}(G)) \subseteq(f(G))^{\text {bso }}$, for every $G \subseteq X$.

Theorem 3.4. Let $f:\left(X, \tau, \preceq_{1}\right) \rightarrow\left(Y, \theta, \preceq_{2}\right)$ be a map. Where $\left(X, \tau, \preceq_{1}\right)$ is a topological ordered space and $\left(Y, \theta, \preceq_{2}\right)$ is a supra topological ordered space. Then:

(i) $f$ is I-supra closed iff $(f(G))^{i s c l} \subseteq f(\operatorname{cl}(G))$, for any $G \subseteq X$.

(ii) $f$ is D-supra closed iff $(f(G))^{d s c l} \subseteq f(c l(G))$, for any $G \subseteq X$.

(iii) $f$ is B-supra closed iff $\left.(f(G))^{\text {bscl }}\right) \subseteq f(c l(G))$, for any $G \subseteq X$.

Proof. (i) ' $\Rightarrow^{\prime}$ Consider $f$ is an I-supra closed map. Then $f(c l(G))$ is an increasing supra closed subset of $Y$. Since $f(G) \subseteq f(c l(G))$, then $(f(G))^{i s c l} \subseteq f(c l(G))$. $' \Leftarrow^{\prime}$ Consider $B$ is a closed subset of $X$. Then $f(B) \subseteq(f(B))^{i s c l} \subseteq f(c l(B))=$ $f(B)$. Therefore $f(B)=(f(B))^{i s c l}$ is an increasing supra closed set. Thus $f$ is an I-supra closed map.

The proof of (ii) and (iii) are similar to (i). 
Theorem 3.5. Let $f:\left(X, \tau, \preceq_{1}\right) \rightarrow\left(Y, \theta, \preceq_{2}\right)$ be a bijective map. Where $\left(X, \tau, \preceq_{1}\right.$ ) is a topological ordered space and $\left(Y, \theta, \preceq_{2}\right)$ is a supra topological ordered space. Then:

(i) $f$ is I-supra open iff $f$ is D-supra closed.

(ii) $f$ is D-supra open iff $f$ is I-supra closed.

(iii) $f$ is B-supra open iff $f$ is B-supra closed.

Proof. (i) ' $\Rightarrow^{\prime}$ Let $f$ be I-supra open and $G$ be a closed subset of $X$. Then $G^{c}$ is open. Since $f$ is bijective, then $f\left(G^{c}\right)=(f(G))^{c}$ which is increasing supra open. Therefore $f(G)$ is a decreasing supra closed subset of $Y$. Thus $f$ is a D-supra closed map.

$' \Leftarrow^{\prime}$ let $f$ be a D-supra closed and $B$ be an open subset of $X$. Then $B^{c}$ is closed. Since $f$ is bijective, then $f\left(B^{c}\right)=(f(B))^{c}$ is decreasing supra closed. Therefore $f(B)$ is an increasing supra open subset of $Y$. Thus $f$ is an I-supra closed map.

The proof of (ii) and (iii) are similar to (i).

Theorem 3.6. Consider $\left(X, \tau, \preceq_{1}\right),\left(Y, \mu, \preceq_{2}\right),\left(Z, \sigma, \preceq_{3}\right)$ are topological ordered spaces and let $\nu$ be an associated supra topology with $\sigma$.

(i) If $f: X \rightarrow Y$ is an open map and $g: Y \rightarrow Z$ is an I-supra (D-supra, B-supra) open map, then $g \circ f$ is an I-supra (D-supra, B-supra) open map.

(ii) If $f: X \rightarrow Y$ is an closed map and $g: Y \rightarrow Z$ is an I-supra (D-supra, B-supra) closed map, then $g \circ f$ is an I-supra (D-supra, B-supra) closed map.

Proof. It is clear .

Theorem 3.7. Consider $\left(X, \tau, \preceq_{1}\right),\left(Y, \mu, \preceq_{2}\right),\left(Z, \sigma, \preceq_{3}\right)$ are topological ordered spaces and let $\nu$ be an associated supra topology with $\sigma$. If $g \circ f$ is I-supra (D-supra, $B$-supra) open and $f: X \rightarrow Y$ is surjective continuous, then $g: Y \rightarrow Z$ is I-supra (D-supra, B-supra) open.

Proof. Consider $g \circ f$ is I-supra open and let $G$ is an open subset of $Y$. Then $f^{-1}(G)$ is an open subset of $X$. Since $g \circ f$ is an I-supra open and $f$ is surjective, then $(g \circ f)\left(f^{-1}(G)\right)=g(G)$ is an I-supra open subset of $Z$. Therefore $g$ is an I-supra open map.

A similar proof can be given for the case between parentheses.

Theorem 3.8. Consider $\left(X, \tau, \preceq_{1}\right),\left(Y, \theta, \preceq_{2}\right),\left(Z, \mu, \preceq_{3}\right)$ are topological ordered spaces and let $\nu$ and $\sigma$ be associated supra topologies with $\theta$ and $\mu$, respectively. If $g \circ f:\left(X, \tau, \preceq_{1}\right) \rightarrow\left(Z, \mu, \preceq_{3}\right)$ is a closed map and $g:\left(Y, \theta, \preceq_{2}\right) \rightarrow\left(Z, \mu, \preceq_{3}\right)$ is $I$ supra (resp. D-supra, B-supra) continuous injective, then $f:\left(X, \tau, \preceq_{1}\right) \rightarrow\left(Y, \theta, \preceq_{2}\right)$ is D-supra (resp. I-supra, B-supra) closed. 
Proof. When $g$ is I-supra continuous, let $G$ be a closed subset of $X$. Then $(g \circ f)(G)$ is a closed subset of $Z$. Since $g$ is an injective and I- supra continuous map, then $g^{-1}(g \circ f)=f(G)$ is a D-supra closed subset of $Y$. Therefore $f$ is a D-supra closed map.

A similar proof can be given for the cases between parentheses.

Theorem 3.9. Consider $f:\left(X, \tau, \preceq_{1}\right) \rightarrow\left(Y, \theta, \preceq_{2}\right)$ is a bijective map and let $\theta^{\star}$ be an associated supra topology with $\theta$. Then:

(i) $f$ is I-supra (resp. D-supra, B-supra) open iff $f^{-1}$ is I-supra (resp. D-supra, B-supra) continuous.

(ii) $f$ is D-supra (resp. I-supra, B-supra) closed iff $f^{-1}$ is I-supra (resp. D-supra, B-supra) continuous.

Proof. (i) We prove (i) when $f$ is a B-supra open, and the other cases are proved similarly.

$' \Rightarrow^{\prime}$ Let $f$ be a B-supra open map and $G$ be an open subset of $X$. Then $\left(f^{-1}\right)^{-1}(G)=f(G)$ is a balancing supra open subset of $Y$. Therefore $f^{-1}$ is a B-supra continuous.

$' \Leftarrow{ }^{\prime}$ let $G$ be an open subset of $X$ and $f^{-1}$ be a B-supra continuous. Then $f(G)=\left(f^{-1}\right)^{-1}(G)$ is a balancing supra open subset of $Y$. Therefore $f$ is B-supra open.

(ii) Similarly, one can prove (ii).

Theorem 3.10. Consider $\left(X, \tau, \preceq_{1}\right)$ is a topological ordered space and $\left(Y, \theta, \preceq_{2}\right)$ is a supra topological ordered space. Let a bijective map $f: X \rightarrow Y$ be I-supra open and order preserving (D-supra closed and order preserving). If $\left(X, \tau, \preceq_{1}\right)$ is a lower $T_{1}$-ordered space, then $\left(Y, \theta, \preceq_{2}\right)$ is a strong lower supra $T_{1}$-ordered space.

Proof. When a map $f$ be I-supra open.

For each $x, y \in Y$ such that $x \npreceq_{2} y$, there exist $a, b \in X$ such that $a=f^{-1}(x), b=$ $f^{-1}(y)$. Since $f$ is an order preserving map, then $a \npreceq_{1} b$. Since $\left(X, \tau, \preceq_{1}\right)$ is a lower $T_{1}$-ordered space, then there exists an increasing neighborhood $W$ in $X$ such that $a \in W$ and $b \notin W$. Therefore there exists an open set $G$ such that $a \in G \subseteq W$. Thus $x \in f(G)$ which is an increasing supra open and $y \notin f(G)$. Hence, $\left(Y, \theta, \preceq_{2}\right)$ is a strong lower supra $T_{1}$-ordered space.

The proof when a map $f$ be I-supra closed is achieved similarly from the equivalence between I-supra open and D-supra closed, when $f$ is bijective.

Theorem 3.11. Consider $\left(X, \tau, \preceq_{1}\right)$ is a topological ordered space and $\left(Y, \theta, \preceq_{2}\right)$ is a supra topological ordered space. Let a bijective map $f: X \rightarrow Y$ be D-supra open and order preserving (I-supra closed and order preserving). If $\left(X, \tau, \preceq_{1}\right)$ is an upper $T_{1}$-ordered space, then $\left(Y, \theta, \preceq_{2}\right)$ is a strong upper supra $T_{1}$-ordered space. 
Proof. The proof is similar to that of Theorem 3.10.

Theorem 3.12. Consider $\left(X, \tau, \preceq_{1}\right)$ is a topological ordered space and $\left(Y, \theta, \preceq_{2}\right)$ is a supra topological ordered space. Let a bijective map $f: X \rightarrow Y$ be B-supra open and order preserving (B-supra closed and order preserving). If $\left(X, \tau, \preceq_{1}\right)$ is a $T_{i}$-ordered space, then $\left(Y, \theta, \preceq_{2}\right)$ is a strong supra $T_{i}$-ordered space for $i=0,1,2$.

Proof. When a map $f$ be I-supra open and $i=2$.

For all $x, y \in Y$ such that $x \npreceq_{2} y$, there are $a, b \in X$ such that $a=f^{-1}(x), b=$ $f^{-1}(y)$. Since $f$ is an order preserving, then $a \npreceq_{1} b$. Since $\left(X, \tau, \preceq_{1}\right)$ is a $T_{2^{-}}$ ordered space, then there exist disjoint neighborhoods $W_{1}$ and $W_{2}$ of $a$ and $b$ respectively such that $W_{1}$ is increasing and $W_{2}$ is decreasing. Therefore there are disjoint open sets $G$ and $H$ such that $a \in G \subseteq W_{1}$ and $b \in H \subseteq W_{2}$. Thus $x \in f(G)$ which is an increasing supra open, $y \in f(H)$ which is a decreasing supra open and $f(G) \bigcap f(H)=\varnothing$. Thus $\left(Y, \theta, \preceq_{2}\right)$ is a strong supra $T_{2}$-ordered space.

In a similar way, we can prove theorem in the cases of $i=0,1$.

The proof when a map $f$ be B-supra closed is achieved similarly from the equivalence between B-supra open and B-supra closed, when $f$ is bijective.

Theorem 3.13. Consider $\left(X, \tau, \preceq_{1}\right)$ is a topological ordered space, $\left(Y, \theta, \preceq_{2}\right)$ is a supra topological ordered space and bijective map $f: X \rightarrow Y$ is supra open such that $f$ and $f^{-1}$ are order preserving. If $\left(X, \tau, \preceq_{1}\right)$ is a $T_{i}$-ordered (strong $T_{i}$-ordered) space, then $\left(Y, \theta, \preceq_{2}\right)$ is a supra $T_{i}$-ordered (strong supra $T_{i}$-ordered) space for $i=$ $0,1,2$.

Proof. When $i=2$, for all $x, y \in Y$ such that $x \npreceq_{2} y$, there exist $a, b \in X$ such that $a=f^{-1}(x), b=f^{-1}(y)$. Since $f$ is an order preserving, then $a \npreceq_{1} b$. Since $\left(X, \tau, \preceq_{1}\right)$ is a $T_{2}$-ordered space, then there exist disjoint increasing neighborhood $W_{1}$ of $a$ and decreasing neighborhood $W_{2}$ of $b$ such that $a \in G \subseteq W_{1}$ and $b \in$ $H \subseteq W_{2}$. Since $f$ is a bijective supra open and $f^{-1}$ is an order preserving, then $f(G) \subseteq f\left(W_{1}\right)$ which is an increasing supra neighborhood of $x, f(H) \subseteq f\left(W_{2}\right)$ which is a decreasing supra neighborhood of $y$ and $f\left(W_{1}\right) \cap f\left(W_{2}\right)=\varnothing$. Therefore $\left(Y, \theta, \preceq_{2}\right)$ is a strong supra $T_{2}$-ordered space.

Similarly, one can prove theorem in the cases of $i=0,1$.

A similar proof can be given for the case between parentheses.

Theorem 3.14. Consider $\left(X, \tau, \preceq_{1}\right)$ is a topological ordered space and $\left(Y, \theta, \preceq_{2}\right)$ is a supra topological ordered space. Let $f: X \rightarrow Y$ be a bijective supra open map such that $f$ and $f^{-1}$ are order preserving. If $\left(X, \tau, \preceq_{1}\right)$ is a $T_{i}$-ordered (a strong $T_{i}$-ordered) space, then $\left(Y, \theta, \preceq_{2}\right)$ is a supra $T_{i}$-ordered (a strong supra $T_{i}$-ordered) space for $i=0,1,2$.

Proof. The proof is similar to that of Theorem 3.13. 


\section{Supra homeomorphism maps in supra topological ordered spaces}

In this section, we introduce the concepts of I-supra homeomorphism, D-supra homeomorphism and B-supra homeomorphism maps in supra topological ordered spaces and study many of their properties.

Definition 4.1. Let $(X, \tau),(Y, \theta)$ be two topological spaces and $\tau^{\star}, \theta^{\star}$ are associated supra topologies with $\tau$ and $\theta$, respectively. A bijective map $g: X \rightarrow Y$ is called supra homeomorphism if it is supra continuous and supra open.

Theorem 4.1. Consider $(X, \tau),(Y, \theta)$ are topological spaces, $\tau^{\star}$ and $\theta^{\star}$ are associated supra topology with $\tau$ and $\theta$, respectively. A bijective map $g: X \rightarrow Y$ is supra homeomorphism iff $f(\operatorname{cl}(E)) \subseteq \operatorname{cl}(f(E))$ and $\operatorname{scl}(f(E)) \subseteq f(\operatorname{cl}(E))$ for every $E \subseteq X$

Proof. It is clear.

Definition 4.2. Let $\left(X, \tau, \preceq_{1}\right),\left(Y, \theta, \preceq_{2}\right)$ be two topological ordered spaces and $\tau^{\star}, \theta^{\star}$ are associated supra topologies with $\tau$ and $\theta$, respectively. A bijective map $g: X \rightarrow Y$ is called I-supra (resp. D-supra, B-supra) homeomorphism if it is I-supra continuous and I-supra open (resp. D-supra continuous and D-supra open, B-supra continuous and B-supra open).

Remark 4.1. (i) Every I-supra (D-supra, B-supra) homeomorphism map is supra homeomorphism.

(ii) Every B-supra homeomorphism map is I-supra homeomorphism and D-supra homeomorphism.

The following two examples illustrate that a supra homeomorphism (resp. Dsupra homeomorphism) map need not be I-supra homeomorphism or D-supra homeomorphism or B-supra homeomorphism (resp. B-supra homeomorphism) map.

Example 4.1. Let the topology $\tau=\{\varnothing, X,\{a, b\}\}$ on $X=\{a, b, c\}$, the supra topology associated with $\tau$ be $\tau^{\star}=\{\varnothing, X,\{a, b\},\{a, c\}\}$ and the partial relations $\preceq_{1}=\triangle \bigcup\{(c, a)\}$ and $\preceq_{2}=\triangle \bigcup\{(b, c)\}$ on $X$. The identity map $f:\left(X, \tau, \preceq_{1}\right) \rightarrow\left(X, \tau, \preceq_{2}\right)$ is a supra homeomorphism map but not $\mathrm{I}(\mathrm{D}, \mathrm{B})$-supra homeomorphism map.

Example 4.2. We replace only the two partial order relations in Example 4.1 by $\preceq=$ $\triangle \bigcup\{(a, c)\}$. Then the map $f$ is $\mathrm{D}$-supra homeomorphism, but not B-supra homeomorphism.

The relationships among types of supra homeomorphism maps are illustrated in the 
following Figure.

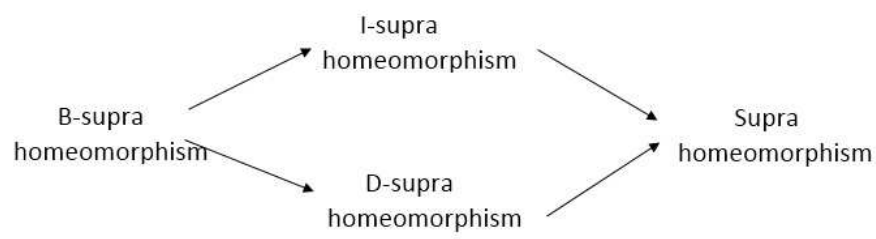

FIG. 4.1: The relationships among types of supra homeomorphism maps

Theorem 4.2. Let a map $f:\left(X, \tau, \preceq_{1}\right) \rightarrow\left(Y, \theta, \preceq_{2}\right)$ be bijective and I-supra continuous. Then the following statements are equivalent:

(i) $f$ is I-supra homeomorphism;

(ii) $f^{-1}$ is I-supra continuous;

(iii) $f$ is D-supra closed.

Proof. (i) $\Rightarrow$ (ii) Let $G$ be an open subset of $X$. Then $\left(f^{-1}\right)^{-1}(G)=f(G)$ is an increasing supra open set in $Y$. Therefore $f^{-1}$ is I-supra continuous.

(ii) $\Rightarrow$ (iii) Let $G$ be a closed subset of $X$. Then $G^{c}$ is an open subset of $X$ and $\left(f^{-1}\right)^{-1}\left(G^{c}\right)=f\left(G^{c}\right)=(f(G))^{c}$ is an increasing supra open set in $Y$. Therefore $f(G)$ is a decreasing supra closed subset of $Y$. Thus $f$ is D-supra closed.

(iii) $\Rightarrow$ (i) Let $G$ be an open subset of $X$. Then $G^{c}$ is a closed set and $f\left(G^{c}\right)=$ $(f(G))^{c}$ is D-supra closed. Therefore $f(G)$ is an I-supra open subset of $Y$. Thus $f$ is I-supra open. Hence $f$ is an I-supra homeomorphism map.

In a similar way, one can prove the following two theorems.

Theorem 4.3. Let a map $f:\left(X, \tau, \preceq_{1}\right) \rightarrow\left(Y, \theta, \preceq_{2}\right)$ be bijective and D-supra continuous. Then the following statements are equivalent:

(i) $f$ is D-supra homeomorphism;

(ii) $f^{-1}$ is D-supra continuous;

(iii) $f$ is I-supra closed.

Theorem 4.4. Let a map $f:\left(X, \tau, \preceq_{1}\right) \rightarrow\left(Y, \theta, \preceq_{2}\right)$ be bijective and B-supra continuous. Then the following statements are equivalent:

(i) $f$ is B-supra homeomorphism;

(ii) $f^{-1}$ is B-supra continuous; 
(iii) $f$ is B-supra closed.

Theorem 4.5. Consider $\left(X, \tau, \preceq_{1}\right),\left(Y, \theta, \preceq_{2}\right)$ are two topological ordered spaces, $\tau^{\star}$ and $\left.\theta^{\star}\right)$ are associated supra topologies with $\tau$ and $\theta$, respectively. Let $f: X \rightarrow Y$ be a supra homeomorphism map such that $f$ and $f^{-1}$ are order preserving. If $X(Y)$ is a $T_{i}$-ordered space, then $Y(X)$ is a supra $T_{i}$-ordered space for $i=0,1,2$.

Proof. (i) Let $\left(X, \tau, \preceq_{1}\right)$ be a $T_{i}$-ordered space, then by Theorem $2.5,\left(Y, \theta, \preceq_{2}\right)$ is a supra $T_{i}$-ordered space for $i=0,1,2$.

(ii) Let $\left(Y, \theta, \preceq_{2}\right)$ be a $T_{i}$-ordered space, then by Theorem $3.13,\left(X, \tau, \preceq_{1}\right)$ is a supra $T_{i}$-ordered space for $i=0,1,2$.

\section{Conclusion}

In the present paper, the concepts of increasing supra continuous (open, closed, homeomorphism) maps, decreasing supra continuous (open, closed, homeomorphism) maps and balancing supra continuous (open, closed, homeomorphism) maps are given and studied. The sufficient conditions for maps to preserve some of separation axioms (which introduced in [1], [3] and [6]) are determined. In particular, we investigate the equivalent conditions for each concept and present their characterizations. Apart from that, we point out the relationships among them with the help of illustrative examples. We plan to generalize the given concepts depending on a notion of $R$-open sets [2]. In the end, the presented concepts in this paper are a fundamental background for studying several topics in supra topological ordered spaces.

\section{R E F E R E N C E S}

1. P. DAS, Separation axioms in ordered spaces, Soochow Journal of Mathematics, 30 (4) (2004) 447-454.

2. M. E. El-Shafei, M. Abo-elhamayel and T. M. Al-shami, On supra $R$-open sets and some applications on topological spaces, Journal of Progressive Research in Mathematics, 8 (2) (2016) 1237-1248.

3. M. E. El-Shafei, M. Abo-elhamayel and T. M. Al-Shami, Strong separation axioms in supra topological ordered spaces, Submitted.

4. M. K. R. S. V. Kumar, Homeomorphism in topological ordered spaces, Acta Ciencia Indian, XXVIII(M)(1)(2012) 67-76.

5. A. S. Mashhour, A. A. Allam, F. S. Mahmoud and F. H. Khedr, On supra topological spaces, Indain J. Pure Appl. Math, 14 (4) (1983) 502-510.

6. S. D. MCCARTAn, Separation axioms for topological ordered spaces, Mathematical Proceedings of the Cambridge Philosophical Society, 64 (1986) 965-973.

7. L. Nachbin, Topology and ordered, D. Van Nostrand Inc. Princeton, New Jersey, (1965). 
8. K. K. RaO And R. Chudamani, $\alpha$-homeomorphism in topological ordered spaces, International Journal of Mathematical Sciences, Technology and Humanities, 52 (2012) 541-560.

9. K. K. RaO And R. Chudamani, $\beta$-homeomorphism in topological ordered spaces, International Journal of Mathematical and Engineering, 182 (2012) 1734-1755.

10. K. K. Rao and R. Chudamani, Pre-homeomorphism in topological ordered spaces, International Journal of Mathematical Sciences, Technology and Humanities, 41 (2012) 408-421.

11. K. K. Rao and R. Chudamani, Semi-homeomorphism in topological ordered spaces, International Journal of Mathematical Sciences, Technology and Humanities, 39 (2012) 383-397.

Mohamed Abo-elhamayel

Department of Mathematics

Mansoura University - Mansoura - Egypt

maboelhamayle@mans.edu.eg

Tareq M. Al-shami

Department of Mathematics

Sana'a University - Sana'a - Yemen

tareqalshami83@gmail.com 